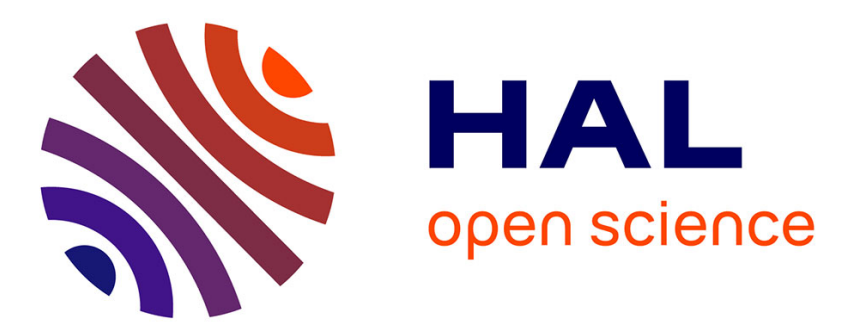

\title{
THE ASYMMETRY OF THE COEXISTENCE CURVE IN LIQUID-VAPOR PHASE TRANSITIONS ; DATA ANALYSIS
}

P. Damay, F. Leclercq

\section{- To cite this version:}

P. Damay, F. Leclercq. THE ASYMMETRY OF THE COEXISTENCE CURVE IN LIQUID-VAPOR PHASE TRANSITIONS; DATA ANALYSIS. Journal de Physique IV Proceedings, 1991, 01 (C5), pp.C5-83-C5-90. 10.1051/jp4:1991510 . jpa-00250634

\section{HAL Id: jpa-00250634 https://hal.science/jpa-00250634}

Submitted on 1 Jan 1991

HAL is a multi-disciplinary open access archive for the deposit and dissemination of scientific research documents, whether they are published or not. The documents may come from teaching and research institutions in France or abroad, or from public or private research centers.
L'archive ouverte pluridisciplinaire HAL, est destinée au dépôt et à la diffusion de documents scientifiques de niveau recherche, publiés ou non, émanant des établissements d'enseignement et de recherche français ou étrangers, des laboratoires publics ou privés. 


\title{
THE ASYMMETRY OF THE COEXISTENCE CURVE IN LIQUID-VAPOR PHASE TRANSITIONS; DATA
} ANALYSIS

\author{
P. DAMAY and F. LECLERCQ \\ Laboratoire de Chimie-Physique, CNRS at HEI, 13 ne de Toul, F-59046 Lille cedex, France
}

\begin{abstract}
The usual way to calculate the critical parameters of a coexistence curve is to separate the coexistence curve from the diameter, taking advantage of the symmetry properties of the diameter. We showed that this procedure presents several drawbacks, the most severe being that the critical concentration may not be obtained with accuracy from the fits.

A new computing way able to determine from a single fit all the parameters of the coexistence curve is presented.

Two of the several advantages of using the new procedure are:

- the divergence of the diameter is obtained with a better precision that any of the two step method.

- the coupling between all the parameters, including the important complying between $\mathrm{A}_{1-\alpha} \mathrm{t}^{1-\alpha}$ and $\rho_{c}$, may be analyzed in detail.
\end{abstract}

\section{I-Introduction and formalism.}

The law of rectilinear diameter, formulated by Cailletet and Matthias $/ 1 /$, is one of the more ancient classical law of the physics of liquids. The law states that the mean density of a system undergoing a liquid-vapor phase transition is a linear function of temperature

$$
\rho_{\mathrm{d}=1 / 2}\left(\rho_{1}+\rho_{\mathrm{v}}\right)
$$

In modern theories of phase transition, liquid-vapor equilibria belong to the universality class of Ising-like or hole-particle models. These theories are based on strict symmetry conditions which are never met in real systems; for example a strict symmetry implies $\rho_{d}=\rho_{c}$ instead of Equ.1( $\rho_{c}$, the critical concentration). There are several ways to overturn the symmetry problem. B.Widom et J.S.Rowlinson $/ 2$ / found a solution when they showed that the diameter was a line of symmetry; the equation of the coexistence curve may formally be separated into two parts, a first term $\left(\rho_{l}-\rho_{v}\right)$ giving the general shape of the coexistence curve is symmetrical with respect to the line of symmetry; the second term $\left(\rho_{1}+\rho_{v}\right)$ accounts for the asymmetry and is not universal.

There are now numerous experimental evidences that the Cailletet-Matthias law is not exact near the critical point $/ 3 /$. Several models showed that the diameter could diverge as a function of $t^{1-\alpha}$, with 
$t=1-T / T_{c}$ is the reduced temperature and $\alpha$ is the index which describes the singularity of the heat capacity $C_{v}$. Furthermore, an other deviation from Equ.1 proportional to $t 2 \beta$ may also be observed if the appropriate order parameter is not chosen.

Bringing everything together, the asymmetric coexistence curve may be defined by two equations

$$
\begin{aligned}
& \Delta_{\rho}=\rho_{1}-\rho_{v}=2 B_{0} t^{\beta}\left(1+B_{1} t^{\Delta_{1}}+B_{2} t^{\Delta_{2}}+\ldots\right) \\
& \rho_{d}=\frac{\rho_{1}+\rho_{v}}{2}=\rho_{c}+A_{1-\alpha} t^{1-\alpha}+A_{1} t+A_{2} t^{2 \beta}+\ldots
\end{aligned}
$$

where $\rho$ is the order parameter at the critical point (for example the density)

$\beta$ is the main critical exponent which determines the shape of the coexistence curve

$B_{1} t^{\Delta 1}$ and $B_{2} t^{\Delta 2}$ are the extended scaling correction terms.

$\mathrm{D}_{0} \mathrm{t}$ is the linear diameter (Cailletet and Matthias /1/)

$A_{1-\alpha} t^{1-\alpha}$ is the 1- $\alpha$ divergence which has been predicted theoretically

$\mathrm{A}_{2} \mathrm{t}^{2 \mathrm{~B}}$ is a less interesting divergence which occurs in case of a "wrong choice" of the order parameter (sometimes referred as a spurious $2 \beta$ divergence $/ 4 /$ )

Equations 1 and 2 may formally be regrouped in a single equation:

$$
p=\rho_{c} \pm B_{0} t^{\beta}\left(1+B_{1} t^{\Delta_{1}}+B_{2} t^{\Delta_{2}}+\ldots\right)+A_{1-\alpha} t^{1-\alpha}+A_{1} t+A_{2} t^{2 \beta}+\ldots
$$

where the the sign $"+"$ and $"-"$ are used for $\rho>\rho_{c}$ and $\rho<\rho_{c}$ respectively.

In practice, the parameters of a coexistence curve are always determined separately from the experimental results using equations 1 and 2. There is no a priori objection of using Equ.3. Of course, there does not exist any experimental data set precise enough to separate the three last contributions in Equ.3; thus the routine used in the fit includes only one term $A t^{\lambda}$ to characterize the diameter, $\lambda$ being either 1 or $1-\alpha$ or $2 \beta$. The least squares procedure using Equ. $3^{\prime}$ is not linear; a test is performed at each iteration on every data point to check if $\rho$ is larger or smaller than $\rho_{c}$ in order to choose the appropriate sign of the second term of Equ.3.

$$
\rho=\rho_{c} \pm B_{0} t^{\beta}\left(1+B_{1} t^{\Delta_{1}}+B_{2} t^{\Delta_{2}}+\ldots\right)+A_{\lambda} t^{\lambda}
$$


What may be the pay-off of using a complicated way of performing the calculation with real risks of spurious oscillations or divergence caused by the test $\rho-\rho_{\mathrm{c}}$ ?

1- From the computing point of view, it is found that the results of the fits using Equ. $3^{\prime}$ are as good as that obtained from Equ. 1 and 2.

2- A first important advantage is that the parameters are obtained from experimental points only; there is no need to evaluate the density of the "conjugate phase" by interpolation. This evaluation is generally easy to perform, excepted very near the critical point. For several systems, differences between 0.003 and 0.01 are found on the value of $\beta$ as obtained from the evaluated data points only and the experimental results using Equ. $3^{\prime}$.

3- If the diameter is a line of symmetry as shown from the work of B.Widom and J.S.Rowlinson $/ 2 /$, the parameters in equations 1 and 2 are completely uncoupled. That would be certainly true if the experimental results were exact. In practice all the parameters defining the coexistence curve are more or less coupled, for example the value of the index $\beta$ influences considerably the value of the index $\lambda$. A benefit of using Equ. $3^{\prime}$ is to check the coupling between parameters.

4- There is no mean to determine the critical density from a fit using equation 2 . Thus $\rho_{c}$ must be evaluated manually and entered as a constant in a fit. It is easy to understand that the choice of $\rho_{c}$ influences to a large extend the curvature of the diameter. It has been shown in a previous study on binary mixtures that it was possible to determine the critical concentration with a great accuracy using an equation similar to $3^{\prime}$. In that case the concentration plays the same role as the density for the liquid-vapor phase transition.

5- The critical temperature is generally obtained with a slightly better accuracy using Equ. $3^{\prime}$ than Equ.1; in several instances, i.e. when the quality and number of data points are not sufficient, the critical temperature itself is evaluated before the fit. Even when the critical temperature is obtained from Equ.1, it is always entered as a constant in Equ.2; this reveals an immediate coupling between parameters of equations 1 and 2.

Three examples have been chosen to examine the use of a fit based on Equ.3' on real systems; the first system investigated is Argon; the coexistence curve has been determined by R.K. Teague and C.J. Pings /5/; the two other systems are metallic, Rubidium and Cesium from the Marburg group /6/. 


\section{II-Three examples.}

a- Argon. The coexistence curve has been determined from a measurement of the refractive index along the coexistence curve. The critical values were obtained from a linear least squares fit in a log$\log$ scale $\quad \log \left(\mathrm{n}_{1}-\mathrm{n}_{\mathrm{d}}\right)=\mathrm{A}_{0}+\beta \ln \left(\mathrm{T}_{\mathrm{c}}-\mathrm{T}\right)$. The value obtained by the authors are compared with that found from a fit using Equ.3' with no extended scaling terms and a range of $t=0.20$

\begin{tabular}{l|ll} 
& Teague and Pings /5/ & This work (Equ. 3') \\
\hline $\mathrm{T}_{\mathrm{c}}$ & $150.704(15) \mathrm{K}$ evaluated & $150.09(4) \mathrm{K}$ \\
$\beta$ & $0.3646(66)$ & $0.3526(26)$ \\
$\mathrm{n}_{\mathrm{c}}$ & $1.08587(10)$ & $1.08154(34)$ \\
$\lambda$ & & $0.76(2)$
\end{tabular}

The critical temperature found from the fit differs significantly from the evaluation of Teague and Pings. The critical refractive indices are well outside the error range.

The correlation matrix is the following

\begin{tabular}{l|lllll} 
& $\mathrm{B}_{0}$ & $\beta$ & $\mathrm{T}_{\mathrm{c}}$ & $\rho_{\mathrm{c}}$ & $\lambda$ \\
\hline $\mathrm{B}_{0}$ & 1 & 0.993 & 0.887 & -0.137 & -0.040 \\
$\beta$ & & 1 & 0.852 & -0.132 & -0.039 \\
$\mathrm{~T}_{\mathrm{c}}$ & & & 1 & -0.155 & -0.045 \\
$\rho_{c}$ & & & & 1 & 0.996 \\
$\lambda$ & & & & & 1
\end{tabular}

The parameters are well separated into two groups $B_{0}, T_{c}, \beta$ and $n_{c}, \lambda$ as expected from writing Equ. 1 and 2. The coupling of parameters inside each group is very strong but the groups are weakly coupled to each other. One notices that $\lambda$ is very strongly coupled to the critical density; it means that the value of $\lambda$ is already largely determined if the critical density is evaluated manually. Thus a good evaluation of the index $\lambda$ will be more secure if the fit is performed in one step as indicated in point 4. 
b-Rubidium. Jüngst,Knuth,Hensel /6/ determined the critical parameters from least squares based on Equ. 1 and Equ.2. The results are as follow:

\begin{tabular}{l|ll} 
& Jüngst,Knuth,Hensel /6/ & This work (Equ. 3') \\
\hline$T_{c}$ & $2017 \mathrm{~K}$ evaluated & $2015.92(22) \mathrm{K}$ \\
$\mathrm{B}_{0}$ & 2.45 & $2.26(10)$ \\
$\beta$ & $0.360(10)$ & $0.360(10)$ \\
$\rho_{c}$ & $0.292 \mathrm{~g} / \mathrm{cm}^{3}$ evaluated & $0.2824(31)$ \\
$\lambda$ & $0.86(3)$ & $0.62(10)$
\end{tabular}

Again it is found that the value of the main critical index $\beta$ is not very sensitive to the type of calculation; it was found that $\beta$ is more sensitive to a proper use of extended scaling corrections terms. On the contrary, the values of $\lambda$ are very different, close to $1-\alpha$ in one case and to $2 \beta$ in the other case. This discrepancy may be understood when considering the strong coupling between $\lambda$ and $\rho_{c}$ in the following correlation matrix and the very different evaluations of the critical density.

\begin{tabular}{l|lllll} 
& $\mathrm{B}_{0}$ & $\beta$ & $\mathrm{T}_{\mathrm{c}}$ & $\rho_{\mathrm{c}}$ & $\lambda$ \\
\hline $\mathrm{B}_{0}$ & 1 & 0.986 & 0.693 & -0.382 & -0.464 \\
$\beta$ & & 1 & 0.662 & -0.380 & -0.482 \\
$\mathrm{~T}_{\mathrm{c}}$ & & & 1 & -0.560 & -0.512 \\
$\rho_{\mathrm{c}}$ & & & & 1 & $\mathbf{0 . 9 1 2}$ \\
$\lambda$ & & & & & 1
\end{tabular}

From several fits using different values of the critical density kept constant, it was possible to determine the influence of varying $\rho_{c}$ on the index $\lambda$; the derivative $d \lambda / d \rho_{c}$ is equal to -25 . The difference between the values of $\lambda$ obtained from the two methods is exactly accounted for by this derivative and the different critical densities as seen in figure 1. Again, a manual evaluation of the critical density largely determines the value of the index $\lambda$ obtained from the fit.

It is also noticeable that, in this system, the parameters do not separate into two groups but that there are all rather strongly coupled to each others. 


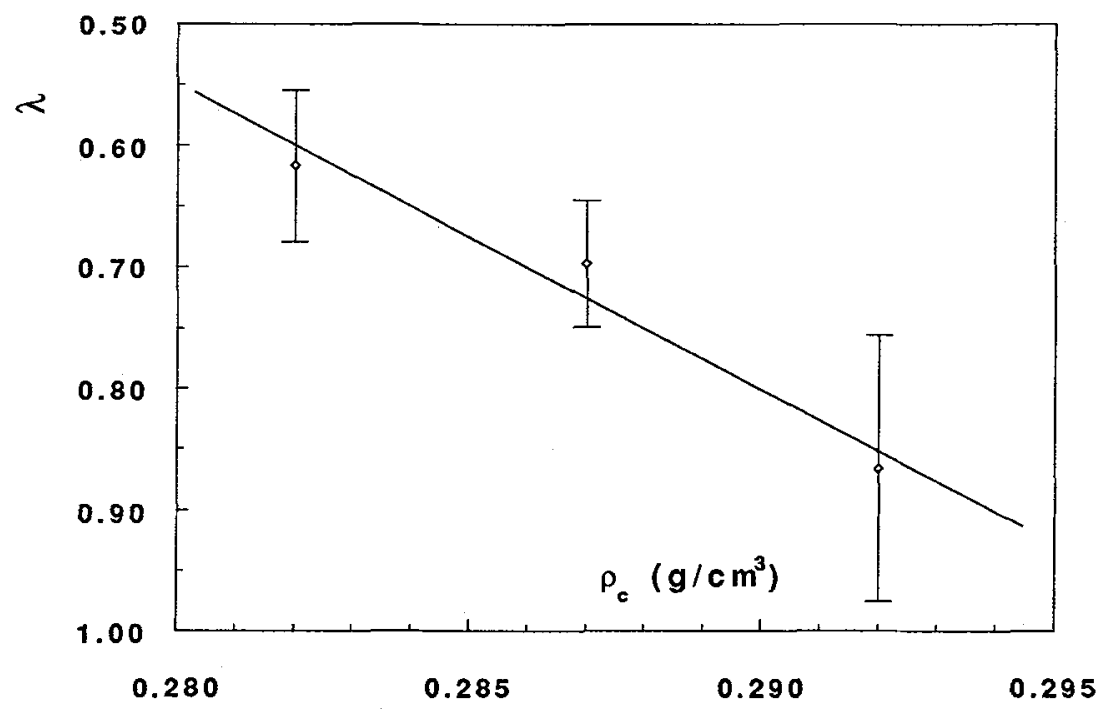

Figure 1. The variation of the critical index $\lambda$ as a function of the critical density $\rho_{c}$

c-Cesium. The experimental data are also from Jüngst,Knuth,Hensel $/ 6 /$. The compared results and the correlation matrix are the followings

\begin{tabular}{l|ll} 
& Jüngst,Knuth,Hensel /6/ & This work (Equ. 3') \\
\hline $\mathrm{T}_{\mathrm{c}}$ & $1924 \mathrm{~K}$ evaluated & $1923.78(4) \mathrm{K}$ \\
$\mathrm{B}_{0}$ & 2.25 & $2.25(7)$ \\
$\beta$ & $0.355(10)$ & $0.353(7)$ \\
$\rho_{c}$ & $0.379 \mathrm{~g} / \mathrm{cm}^{3}$ evaluated & $0.3788(25)$ \\
$\lambda$ & $0.87(3)$ & $0.91(7)$
\end{tabular}




\begin{tabular}{l|lllll} 
& $\mathrm{B}_{0}$ & $\beta$ & $\mathrm{T}_{\mathrm{c}}$ & $\rho_{\mathrm{c}}$ & $\lambda$ \\
\hline $\mathrm{B}_{0}$ & 1 & 0.985 & 0.468 & -0.084 & 0.093 \\
$\beta$ & & 1 & 0.662 & -0.068 & 0.133 \\
$\mathrm{~T}_{\mathrm{c}}$ & & & 1 & -0.132 & -0.015 \\
$\rho_{\mathrm{c}}$ & & & & 1 & $\mathbf{0 . 9 9 5}$ \\
$\lambda$ & & & & & 1
\end{tabular}

As for Argon, the parameters are well separated into two groups with a weak coupling between the groups. Again the parameters $\rho_{c}$ and $\lambda$ are strongly coupled. The values of $\lambda$ found by the two methods are similar because the manual evaluation of the critical density used in the first method is very closed to that found from the fit (Equ $3^{\prime}$ ). Experimental data and calculated curve are reported in figure 2.

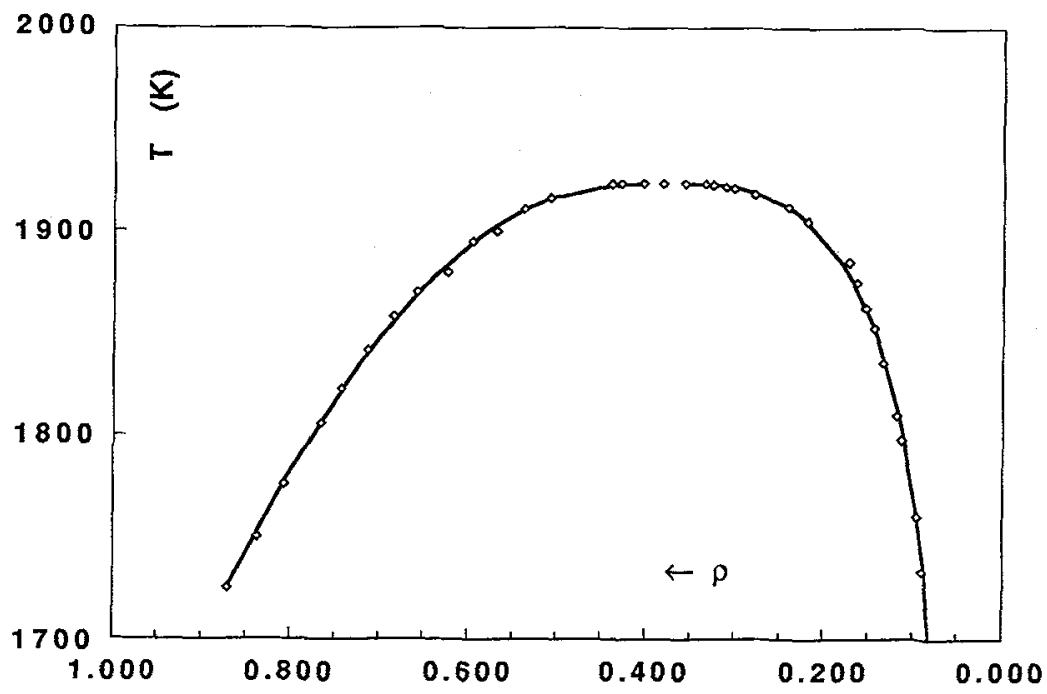

Figure 2. Experimental data $16 /$ and calculated coexistence curve for the liquid-vapor phase transition in liquid cesium. The strong asymmetry of the curve is well accounted for using equation 3'. 


\section{III-Concluding remarks.}

There are several aspects of the work which were not discussed here; for example, the results obtained from the complete equation $3 '$, including extended scaling, are not showed.

The two main points we wanted to emphasize are the followings:

- if a separation in two equations ( 1 and 2 ) is permitted by theory, the errors on experimental data bring a coupling between all the critical parameters. Nevertheless, the strong coupling between parameters of equation 1 and 2 for rubidium is not well understood.

- no parameter should be determined manually, prior to the fit, if one wants to discuss secondary effects as the curvature of the diameter. On the contrary, the main parameters as $\beta$ or $B_{0}$ are very stable and the easier two-steps calculation using Equ. 1 and 2 is possible.

\section{Bibliography.}

/1/ CAILLETET L., and MATHIAS E., C.R.Acad.Sci. 102, 1202 (1886)

12) WIDOM B. and ROWLINSON J.S., J.Chem.Phys. 58, 1670 (1970)

13/ ROWLINSON J.S., Nature 319, 6052 (1986)

14/ SCOTT R.L., in Specialist Periodical Reports, Chemical Thermodynamics, edited by McGlashan, The Chemical Society, London (1978)

15/ TEAGUE R.K. and PINGS C.J., J.Chem.Phys. 48, 4973 (1968)

16/ JUNGST S., KNUTH B. and HENSEL F., Phys.Rev.Lett. 20, 2160 (1985) 